\title{
Hydrogeology and hydrogeochemistry of an alkaline volcanic area: the NE Mt. Meru slope (East African Rift - Northern Tanzania)
}

\author{
G. Ghiglieri ${ }^{1}$, D. Pittalis ${ }^{2}$, G. Cerri ${ }^{3}$, and G. Oggiano ${ }^{3}$ \\ ${ }^{1}$ Department of Earth Sciences, University of Cagliari, Italy, Desertification Research Group - NRD, \\ University of Sassari, Italy \\ ${ }^{2}$ Department of Territorial Engineering, Desertification Research Group - NRD, University of Sassari, Italy \\ ${ }^{3}$ Department of Botanical, Ecological and Geological Sciences, Desertification Research Group - NRD, \\ University of Sassari, Italy
}

Correspondence to: G. Ghiglieri (ghiglieri@unica.it)

Received: 22 July 2011 - Published in Hydrol. Earth Syst. Sci. Discuss.: 1 September 2011

Revised: 27 December 2011 - Accepted: 3 February 2012 - Published: 17 February 2012

\begin{abstract}
The objective of this study is to analyze the geochemical conditions associated with the presence of fluoride $\left(\mathrm{F}^{-}\right)$in the groundwater of an area of Northern Tanzania. The studied aquifers are composed of volcanic rocks such as phonolitic and nephelinitic lavas, basalts, lahars of various ages and mantling ash. Sedimentary rocks consisting of fine-grained alluvial and lacustrine deposits occur as well. Samples collected from springs, borehole and surface water, during two monitoring surveys, were analyzed for the various physico-chemical and isotopic parameters. The geochemical composition of water is typically sodium bicarbonate. High values of $\mathrm{F}^{-}$(up to $68 \mathrm{mgl}^{-1}$ ) were recorded. The highest values of fluoride agreed with the highest values of $\mathrm{pH}$, sodium and bicarbonate. Dissolution of major ions, exchange processes and precipitation of $\mathrm{Ca}^{2+}$ from supersaturated solutions joined with the local permeability and hydraulic gradients, control the fluoride mobilization and the contamination of the area.
\end{abstract}

\section{Introduction}

The pollution of groundwater has become a major environmental issue, particularly where groundwater represents the main source of drinking water. This situation is so common in many lesser developed countries that the security of drinking water supply has been chosen as one of the ten
Millennium Development Goals. The natural contamination of groundwater resulting from its surrounding geological environment can be an important factor determining the quality of drinking water. One of the most severe such natural contaminants is fluoride, especially in the East African Rift Valley, where malformed bones and certain neurological ailments are known to be caused by

Fluorosis, and where the presence of fluoride has some influence on the ecology of the region. Sources of fluoride derives largely from rock minerals, air and seawater, but anthropogenic activity can also make a contribution (Fuge and Andrews, 1988). In Tanzania, for example, the level of fluoride in drinking water frequently exceeds the WHO guideline of $1.5 \mathrm{mg} \mathrm{l}^{-1}$ and, from time to time, even the $4.0 \mathrm{mg} \mathrm{l}^{-1}$ limit set by the Tanzanian Government. The alkaline volcanism in the Rift Valley has been implicated for the high fluoride concentration in the local groundwater (Clarke et al., 1990; Davies, 1996; Deocampo, 2003), but neither the behaviour of fluoride in relation to the rock composition of the aquifer, nor the residence time, nor the concentration of other ions have been widely researched. The present study sought to explore the chemistry of the groundwater in the Arusha region of Tanzania, using a combination of hydrochemical, minero-chemical and isotopic analyses, with a view to developing a set of criteria for identifying low fluoride carrying sources of groundwater (Ghiglieri et al., 2010). 

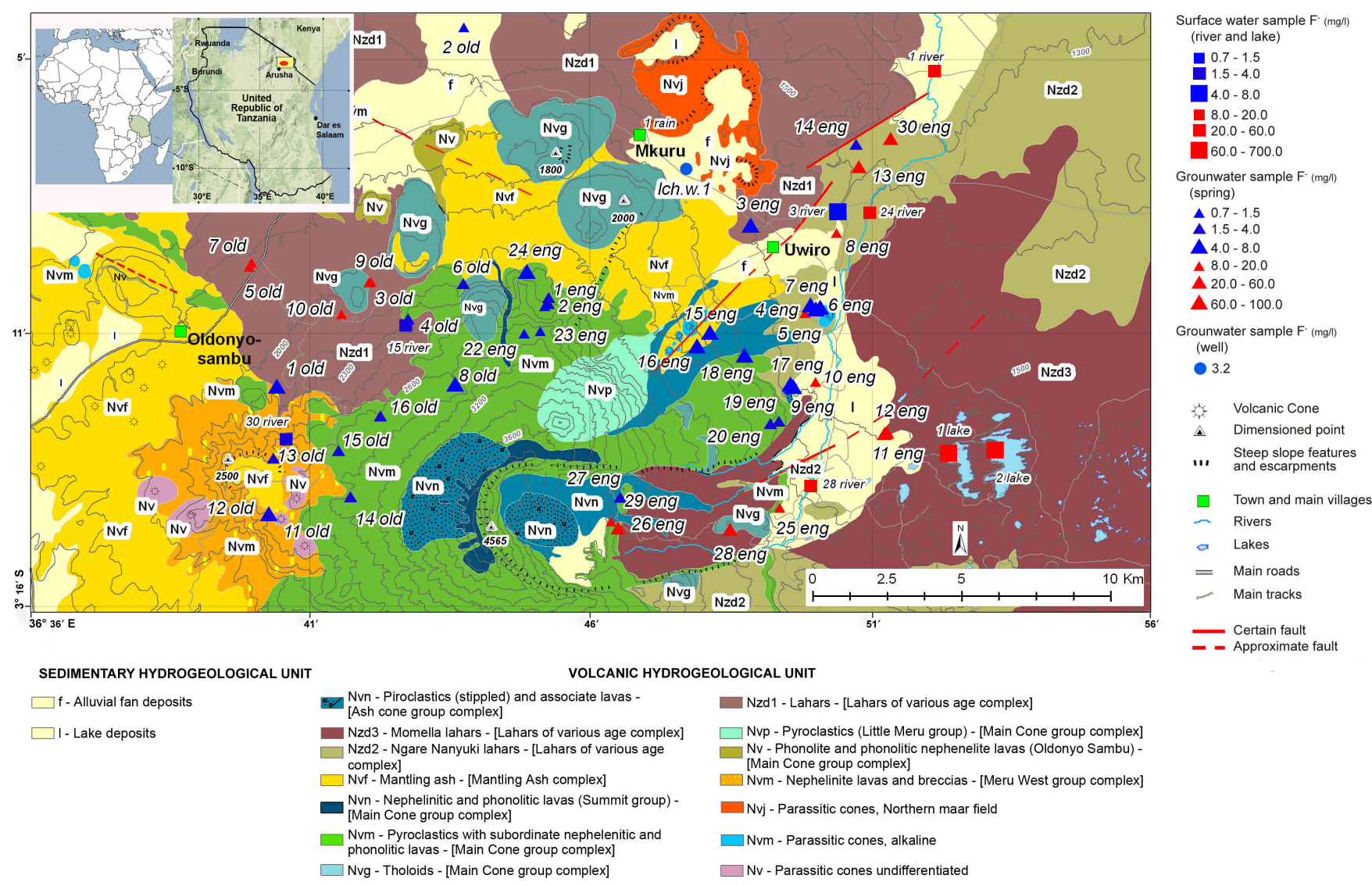

Fig. 1. Study area and related hydrogeological map indicating the location of water points.

\section{Study area and methodology}

\subsection{Study area}

The study area lies within the Meru District, which administratively comprises six divisions, 37 wards and 133 villages (Fig. 1).

It is bounded by Mt. Meru and the Arusha National Park, occupies about $370 \mathrm{~km}^{2}$ and includes nine villages within the Oldonyo Sambu and Ngarenanyuki wards. The area is part of the Maasai Steppe, which extends from Lake Turkana in Kenya to central Tanzania. The natural vegetation is typically savannah. The topography of the study region is dominated by the Mt. Meru volcanic cone of Pleistocene to recent origin. The local climate is temperate Afro-Alpine, with an annual precipitation of $450 \mathrm{~mm}$ (Hijmans et al., 2005) and mean daily temperature minima and maxima of, respectively, $20.6^{\circ} \mathrm{C}$ and $28.5^{\circ} \mathrm{C}$. The rainfall is irregularly distributed between a main wet season from February to mid May (which contributes $\sim 70 \%$ of the annual precipitation), and a minor one from September to November which provides much of the remainder.

\subsection{Geological and hydrogeological setting}

The relationship between the local geology and hydrogeology has been described elsewhere (Ghiglieri et al., 2010). The volcano-sedimentary succession is Cenozoic, with some features dating back to the Miocene-Pliocene, and more recent ones to the Olocene. Volcanic rock dominates, with some recent alluvial deposits. There are no crystalline basement outcrops, rather this material lies at a shallow depth a few kilometers to the north of the study area. Major rift faults are present on the NW margin (Matuginigi and Matisiwi Escarpment). Linear features and benches are commonplace on the flanks of Mt. Meru, and it is highly probable that the early volcanic structure has been block-faulted. In the central area, the faults trend either N-S or NNE-SSW (Uwiro graben), while in the NWsection, the trend is NW-SE (parasitic cone in Lassarkartarta). The two dominant hydrogeological features are the volcanic phonolitic and nephelinitic lavas (as well as basalts, lahars of various ages and mantling ash) and sedimentary material of fine-grained alluvial and lacustrine origin. Groundwater recharge, transmission and discharge are determined by a combination of geomorphology, geology and structural patterns (Ghiglieri et al., 2010), and both shallow and deep circulating groundwater can be 


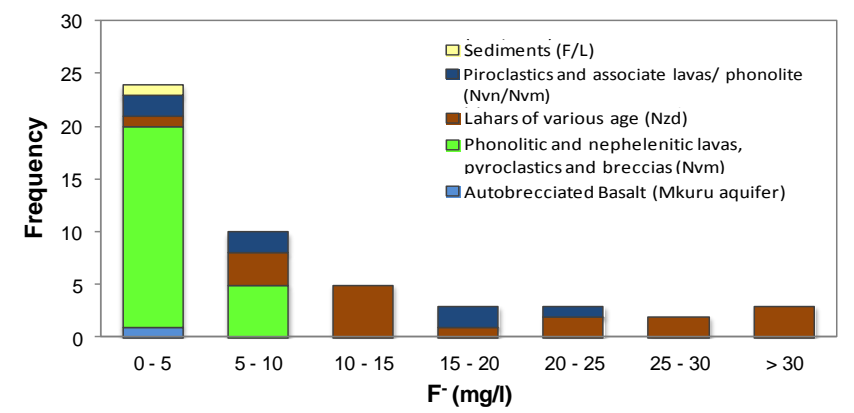

Fig. 2. Stacked vertical bar chart of groundwater samples for the Masika and pre-Masika survey.

distinguished. Shallow groundwater hosted in unconsolidated or semi-consolidated saturated sediments are referred to as local systems. Intermediate and deep groundwater circulation occurs where the permeability of the aquifer and a suficient elevation difference between recharge and discharge area allow deep infiltration. Deep infiltration is also promoted where widespread fracturing and faulting affects the rock.

Where these circumstances prevail, substantial precipitation levels can support productive wells and springs (e.g. the main cone group complex Nvm as reported in the legend of Fig. 1). The groundwater is characterized by a multidirectional flow, dominated by movement from the higher elevation southern part of the region towards the lower lying area in the north. Recharge is via both rainfall infiltration and lateral connections to other hydrogeologic units. The cone-shaped relief generates a general groundwater radial flow, which locally is influenced by facture densities and porosities of the different hydrogeological units (Ghiglieri et al., 2010). The latter affects the Mkuru area in particular, where an aquifer lying in weathered and scoriaceous basalt at a depth of $40-60 \mathrm{~m}$ is fed by groundwater infiltrated from a high elevation area in the main cone group.

\subsection{Material and methods}

\subsubsection{Collection of field data and analytical procedures}

Water was sampled at three different times, determined by a survey conducted in February 2007, with subsequent monitoring (Ghiglieri et al., 2010). A set of 58 sites was established, including 46 springs (30 in the Ngarenanyuki ward and 16 in 5 Oldonyosambu) and six surface water sites (Fig. 1). Portable devices (Hanna Instrument models HI $98130 \mathrm{pH} / \mathrm{EC} / \mathrm{T}$ and HI 93739 photometer), were employed to record $\mathrm{pH}$, electrical conductivity, temperature and fluoride concentration. Two monitoring surveys were implemented, the first in March-April 2007, referred to as Masika, involved 34 samples (25 spring waters, nine surface waters), and the second in January 2008, 10 (pre-Masika) 31 samples (25 spring waters, six surface waters). Two samples

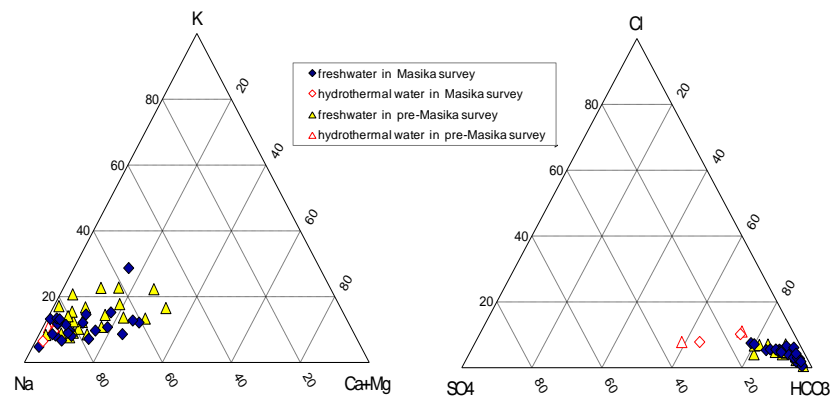

Fig. 3. Ternary plot showing the study area groundwater composition. The $(\mathrm{Ca}+\mathrm{Mg})-\mathrm{Na}-\mathrm{K}$ ternary plot is shown on the left, and the $\mathrm{HCO}_{3}-\mathrm{SO}_{4}-\mathrm{Cl}$ one on the right.

from each site were filtered $(0.45 \mu \mathrm{m})$ into 11 polythene bottles thoroughly pre-washed with distilled water. The samples were transported in low temperature thermal bags and stored under refrigeration.

Standard methods (APHA, 1992) were applied to quantify the presence of major ions, and the analysis of nitrate, nitrite, ammonia and fluoride was performed at the AUWSA laboratory in Arusha. Other chemical determinations were undertaken at the University of Sassari Department of Territorial Engineering. Cation presence $\left(\mathrm{Na}^{+}, \mathrm{K}^{+}, \mathrm{Ca}^{2+}, \mathrm{Mg}^{2+}\right)$ was determined by atomic absorption spectrometry (Perkin Elmer model AAnalist 200), and that of anions $\left(\mathrm{F}^{-}, \mathrm{Cl}^{-}\right.$, $\mathrm{SO}_{4}^{2-}$ ) by ion chromatography (anion column 20 Alltech model allsep anion $7 \mu \mathrm{m}, 100 \mathrm{~mm}$ ). Carbonate and bicarbonate contents were obtained by titration, and silicate by colorimetry. For the sample from spring 26 ENG, elemental analysis was performed by inductively coupled plasma mass spectrometry at the University of Barcelona. The ion balance errors for the analyses were generally within $\pm 5 \%$. The saturation index (hereafter, SI) for fluorite, fluorapatite, calcite, villiaumite and the chemical facies were computed using PHREEQC v2.1 software (Parkhurst and Appelo, 1999) and AQUACHEM v3.1 (SigmaStat software). Isotopic analyses of ${ }^{18} \mathrm{O},{ }^{2} \mathrm{H}$ and ${ }^{3} \mathrm{H}$ were carried out at the CNR Laboratory (Pisa) for the set of Masika samples along with a sample from Ichnusa Well1 collected in January 2008, applying analytical methods described, respectively, by Epstein and Mayeda (1953), Coleman et al. (1982) and the US Department of Energy (1997). Isotope content values $\left(\delta^{18} \mathrm{O}, \delta \mathrm{D}\right)$ are expressed in \%o relative to the Vienna Standard Mean Ocean Water (VSMOW) defined by Craig (1961) with analytical reproducibility within $\pm 0.2 \%$ o for $\delta^{18} \mathrm{O}$ and within $\pm 1 \%$ for $\delta \mathrm{D}$, and ${ }^{3} \mathrm{H}$ concentrations in terms of tritium units (TU) whose analytical reproducibility is reported in Table 3.

Mineralogical analyses were performed on a set of 12 samples at the Department of Botanical, Ecological and Geological Sciences, University of Sassari using a SIEMENS D5000 $\mathrm{X}$-ray diffractometer (Bragg-Brentano geometry) equipped with a $\mathrm{Cu}$ tube and a graphite monochromator on the 
Table 1. Major ion composition of groundwater.

\begin{tabular}{|c|c|c|c|c|c|c|c|c|c|c|c|c|c|c|c|c|c|c|}
\hline \multirow[b]{2}{*}{$\begin{array}{l}\text { ID } \\
\text { sample }\end{array}$} & \multicolumn{2}{|c|}{$T$} & \multicolumn{2}{|c|}{$\mathrm{pH}$} & \multicolumn{2}{|c|}{ Conductivity } & \multirow{2}{*}{$\begin{array}{r}\text { TDS } \\
\text { Jan } \\
2008\end{array}$} & \multicolumn{2}{|c|}{$\mathrm{Ca}^{2+}$} & \multicolumn{2}{|c|}{$\mathrm{Mg}^{2+}$} & \multicolumn{2}{|c|}{$\mathrm{Na}^{+}$} & \multicolumn{2}{|c|}{$\mathrm{K}^{+}$} & \multicolumn{2}{|c|}{$\mathrm{HCO}_{3}^{-}$} & \multirow{2}{*}{$\begin{array}{r}\mathrm{Cl}^{-} \\
\mathrm{Apr} \\
2007\end{array}$} \\
\hline & $\begin{array}{r}\text { Apr } \\
2007\end{array}$ & $\begin{array}{r}\text { Jan } \\
2008\end{array}$ & $\begin{array}{r}\text { Apr } \\
2007\end{array}$ & $\begin{array}{r}\text { Jan } \\
2008\end{array}$ & $\begin{array}{r}\text { Apr } \\
2007\end{array}$ & $\begin{array}{r}\text { Jan } \\
2008\end{array}$ & & $\begin{array}{r}\text { Apr } \\
2007\end{array}$ & $\begin{array}{r}\text { Jan } \\
2008\end{array}$ & $\begin{array}{r}\text { Apr } \\
2007\end{array}$ & $\begin{array}{r}\text { Jan } \\
2008\end{array}$ & $\begin{array}{r}\text { Apr } \\
2007\end{array}$ & $\begin{array}{r}\text { Jan } \\
2008\end{array}$ & $\begin{array}{r}\text { Apr } \\
2007\end{array}$ & $\begin{array}{r}\text { Jan } \\
2008\end{array}$ & $\begin{array}{r}\text { Apr } \\
2007\end{array}$ & $\begin{array}{r}\text { Jan } \\
2008\end{array}$ & \\
\hline 1old & 14,6 & 14,4 & 8,0 & 8,4 & 750 & 790 & 603,08 & 1,15 & 0,72 & 0,27 & 0,26 & 4,26 & 4,78 & 0,92 & 0,64 & 6,14 & 5,86 & 18,05 \\
\hline 2old & 23,6 & n.a. & 6,4 & n.a. & 210 & n.a. & n.a. & 0,41 & n.a. & 0,13 & n.a. & 1,07 & n.a. & 0,47 & n.a. & 1,62 & n.a. & 0,50 \\
\hline 3old & 14,4 & 14,2 & 6,2 & 6,5 & 480 & 470 & 355,13 & 0,35 & 0,07 & 0,02 & 0,02 & 3,09 & 3,22 & 0,71 & 0,52 & 3,24 & 2,75 & 0,14 \\
\hline 4old & 14,2 & 15,9 & 6,4 & 7,2 & 540 & 540 & 410,72 & 0,59 & 0,52 & 0,28 & 0,26 & 3,00 & 3,09 & 0,85 & 0,71 & 3,89 & 3,24 & 0,20 \\
\hline 5old & 17,7 & 17,2 & 7,4 & 7,4 & 610 & 640 & 514,34 & 0,27 & 0,14 & 0,04 & 0,04 & 4,35 & 4,61 & 0,87 & 0,74 & 4,39 & 4,24 & 0,21 \\
\hline 6old & 13,1 & 14,7 & 6,0 & 6,0 & 690 & 660 & 587,75 & 0,36 & 0,24 & 0,20 & 0,19 & 5,22 & 5,22 & 0,50 & 0,42 & 6,29 & 5,82 & 0,25 \\
\hline 8old & 10,7 & 12,3 & 6,7 & 7,0 & 190 & 190 & 185,83 & 0,07 & 0,04 & 0,01 & 0,01 & 1,35 & 1,39 & 0,24 & 0,14 & 1,46 & 1,26 & 0,13 \\
\hline 10old & 14,5 & 14,5 & 7,2 & 7,1 & 480 & 470 & 370,05 & 0,19 & 0,10 & 0,03 & 0,03 & 3,18 & 3,26 & 0,54 & 0,47 & 3,16 & 2,79 & 0,06 \\
\hline 13old & 14,1 & 15,7 & 7,3 & 7,1 & 580 & 540 & 442,59 & 0,39 & 0,30 & 0,17 & 0,13 & 3,52 & 3,22 & 1,21 & 0,64 & 4,98 & 4,00 & 0,15 \\
\hline 16old & 11,9 & 16,0 & 7,2 & 7,0 & 200 & 170 & 155,82 & 0,24 & 0,14 & 0,08 & 0,04 & 1,26 & 0,67 & 0,47 & 0,35 & 1,79 & 1,22 & 0,15 \\
\hline leng & 18,2 & 15,4 & 7,2 & 7,1 & 620 & 600 & 513,39 & 0,67 & 0,41 & 0,23 & 0,41 & 5,22 & 4,35 & 0,59 & 0,42 & 5,15 & 4,91 & 0,07 \\
\hline 2eng & 15,2 & n.a. & 5,9 & n.a. & 490 & n.a. & n.a. & 0,79 & n.a. & 0,42 & n.a. & 2,52 & n.a. & 0,59 & n.a. & 3,72 & n.a. & 0,14 \\
\hline 2beng & n.a. & 15,5 & n.a. & 7,1 & n.a. & 770 & 632,89 & n.a. & 1,27 & n.a. & 0,35 & n.a. & 4,35 & n.a. & 0,89 & n.a. & 5,99 & n.a. \\
\hline 3eng & 23,6 & 24,0 & 7,4 & 7,3 & 720 & 670 & 577,40 & 0,49 & 0,39 & 0,14 & 0,12 & 4,70 & 5,22 & 0,77 & 0,63 & 6,22 & 5,72 & 0,17 \\
\hline 5eng & 22,1 & 20,5 & 7,0 & 6,7 & 980 & 650 & 502,14 & 0,75 & 0,37 & 0,31 & 0,16 & 8,26 & 3,91 & 1,10 & 0,63 & 8,25 & 4,53 & 0,62 \\
\hline 8eng & 22,7 & 21,5 & 7,0 & 7,0 & 1220 & 1340 & 945,64 & 0,95 & 0,74 & 0,40 & 0,31 & 5,92 & 9,35 & 1,25 & 0,95 & 7,50 & 8,63 & 0,40 \\
\hline 16eng & 17,0 & 16,5 & 6,4 & 6,3 & 340 & 340 & 285,53 & 0,13 & 0,08 & 0,04 & 0,04 & 2,52 & 2,39 & 0,27 & 0,23 & 2,55 & 2,30 & 0,15 \\
\hline $18 \mathrm{eng}$ & 18,5 & 18,1 & 6,5 & 6,4 & 470 & 450 & 384,42 & 0,24 & 0,20 & 0,12 & 0,10 & 3,18 & 3,22 & 0,36 & 0,36 & 3,53 & 3,04 & 0,20 \\
\hline 19eng & 16,9 & 16,2 & 7,4 & 7,2 & 400 & 390 & 333,91 & 0,21 & 0,14 & 0,05 & 0,05 & 2,78 & 2,70 & 0,45 & 0,38 & 3,16 & 2,77 & 0,18 \\
\hline 22eng & 12,7 & 14,0 & 5,9 & 6,9 & 250 & 230 & 213,87 & 0,55 & 0,31 & 0,28 & 0,16 & 1,30 & 1,17 & 0,43 & 0,25 & 2,06 & 1,55 & 0,12 \\
\hline $24 \mathrm{eng}$ & 18,3 & 17,7 & 7,2 & 7,0 & 1070 & 1170 & 961,22 & 1,30 & 2,05 & 0,49 & 0,48 & 7,83 & 7,18 & 1,20 & 0,95 & 9,83 & 9,93 & 0,08 \\
\hline 26eng & 22,4 & 22,3 & 7,7 & 7,6 & 5070 & 4730 & 3927,60 & 0,40 & 0,67 & 0,27 & 0,94 & 47,85 & 35,67 & 1,20 & 4,60 & 35,12 & 36,60 & 5,35 \\
\hline 27eng & 13,9 & 12,2 & 6,8 & 6,9 & 330 & 320 & 289,63 & 0,03 & 0,03 & 0,00 & 0,01 & 2,04 & 2,57 & 0,20 & 0,14 & 2,08 & 2,39 & 0,06 \\
\hline 28eng & 17,8 & 17,6 & 7,6 & 7,7 & 1500 & 1160 & 896,46 & 0,27 & 0,24 & 0,12 & 0,10 & 9,05 & 8,48 & 2,51 & 1,20 & 9,53 & 7,88 & 0,84 \\
\hline $29 \mathrm{eng}$ & 17,3 & 17,5 & 7,8 & 7,7 & 1390 & 1870 & 1537,35 & 0,08 & 0,04 & 0,02 & 0,05 & 7,92 & 15,01 & 1,69 & 2,35 & 7,96 & 14,04 & 0,70 \\
\hline 30eng & 24,8 & 24,7 & 8,1 & 7,9 & 3740 & 3500 & 2808,44 & 0,60 & 0,34 & 0,29 & 0,24 & 26,75 & 30,45 & 3,43 & 2,25 & 18,72 & 22,29 & 2,59 \\
\hline $\begin{array}{l}\text { Ichnusa } \\
\text { well } 1\end{array}$ & n.a. & 21,5 & n.a. & 6,4 & n.a. & 620 & 526,46 & n.a. & 0,72 & n.a. & 0,23 & n.a. & 3,65 & n.a. & 0,57 & n.a. & 5,15 & n.a. \\
\hline
\end{tabular}

Chemical composition of groundwater in the study area in April (Apr) 2007 and January (Jan) 2008; all concentrations are expressed in meq/l except pH, conductivity ( $\mu \mathrm{S}$ cm ${ }^{-1}$ ), temperature $\left({ }^{\circ} \mathrm{C}\right)$ and $\operatorname{RESIDUE}\left(\mathrm{mg} \mathrm{l}^{-1}\right)$; n.a. $=$ not available. $*$ With $0,0 \mathrm{mg} \mathrm{l}^{-1}$ are represented the values out of instrument sensitivity.

Table 1. Continued.

\begin{tabular}{|c|c|c|c|c|c|c|c|c|c|c|c|c|c|c|c|}
\hline \multirow[b]{2}{*}{$\begin{array}{l}\text { ID } \\
\text { sample }\end{array}$} & \multirow{2}{*}{$\begin{array}{r}\mathrm{Cl}^{-} \\
\mathrm{Jan} \\
2008\end{array}$} & \multicolumn{2}{|c|}{$\mathrm{SO}_{4}^{2-}$} & \multicolumn{2}{|c|}{$\mathrm{NO}_{3}^{-}$} & \multicolumn{2}{|c|}{$\mathrm{NO}_{2}^{-}$} & \multicolumn{2}{|c|}{$\mathrm{NH}_{3}^{*}$} & \multicolumn{2}{|c|}{$\mathrm{F}^{-}$} & \multirow{2}{*}{$\begin{array}{r}\mathrm{SiO}_{2} \\
\mathrm{Apr} \\
2007\end{array}$} & \multirow{2}{*}{$\begin{array}{r}\text { RESIDUE } \\
110^{\circ} \mathrm{C} \\
\text { Jan } \\
2008\end{array}$} & \multirow{2}{*}{$\begin{array}{r}\text { RESIDUE } \\
180^{\circ} \mathrm{C} \\
\text { Jan } \\
2008\end{array}$} & \multirow[t]{2}{*}{$\begin{array}{r}\text { Altitude } \\
\text { (m.a.s.1.) }\end{array}$} \\
\hline & & $\begin{array}{r}\text { Apr } \\
2007\end{array}$ & $\begin{array}{r}\text { Jan } \\
2008\end{array}$ & $\begin{array}{r}\text { Apr } \\
2007\end{array}$ & $\begin{array}{r}\text { Jan } \\
2008\end{array}$ & $\begin{array}{l}\text { Apr } \\
2007\end{array}$ & $\begin{array}{r}\text { Jan } \\
2008\end{array}$ & $\begin{array}{r}\text { Apr } \\
2007\end{array}$ & $\begin{array}{r}\text { Jan } \\
2008\end{array}$ & $\begin{array}{r}\text { Apr } \\
2007\end{array}$ & $\begin{array}{r}\text { Jan } \\
2008\end{array}$ & & & & \\
\hline 1old & 0,27 & 0,28 & 0,31 & 0,05 & 0,23 & 0,004 & 0,003 & 0,001 & 0,001 & 0,23 & 0,25 & 0,803 & 460,00 & 420,00 & 790 \\
\hline 2old & n.a. & 0,17 & n.a. & 0,07 & n.a. & 0,016 & n.a. & 0,002 & n.a. & 0,05 & n.a. & n.a. & n.a. & n.a. & 470 \\
\hline 3old & 0,18 & 0,21 & 0,24 & 0,17 & 0,18 & 0,005 & 0,002 & 0,012 & 0,001 & 0,65 & 0,63 & 0,826 & 260,00 & 220,00 & 540 \\
\hline 4old & 0,20 & 0,22 & 0,23 & 0,34 & 0,44 & 0,007 & 0,006 & 0,002 & 0,001 & 0,13 & 0,10 & 0,879 & 340,00 & 340,00 & 640 \\
\hline 5old & 0,23 & 0,28 & 0,31 & 0,02 & 0,30 & 0,006 & 0,004 & 0,001 & 0,001 & 0,68 & 1,05 & 0,909 & 360,00 & 340,00 & 660 \\
\hline 6old & 0,13 & 0,12 & 0,14 & 0,02 & 0,08 & 0,004 & 0,001 & 0,001 & 0,001 & 0,13 & 0,16 & 1,144 & 380,00 & 360,00 & 190 \\
\hline 8old & 0,06 & 0,04 & 0,04 & 0,02 & 0,03 & 0,005 & 0,002 & 0,007 & 0,001 & 0,24 & 0,27 & 0,848 & 80,00 & 80,00 & 470 \\
\hline 10old & 0,17 & 0,23 & 0,23 & 0,04 & 0,20 & 0,005 & 0,003 & 0,005 & 0,001 & 0,93 & 1,05 & 0,902 & 240,00 & 220,00 & 540 \\
\hline 13old & 0,13 & 0,13 & 0,13 & 0,11 & 0,28 & 0,005 & 0,004 & 0,002 & 0,001 & 0,21 & 0,22 & 0,977 & 360,00 & 340,00 & 170 \\
\hline 16old & 0,05 & 0,05 & 0,04 & 0,02 & 0,20 & 0,007 & 0,003 & 0,003 & 0,001 & 0,08 & 0,11 & 0,492 & 140,00 & 80,00 & 790 \\
\hline 1eng & 0,11 & 0,18 & 0,19 & 0,26 & 0,27 & 0,005 & 0,004 & 0,001 & 0,001 & 0,16 & 0,16 & 0,848 & 380,00 & 380,00 & 600 \\
\hline 2eng & n.a. & 0,15 & n.a. & 0,05 & n.a. & 0,004 & n.a. & 0,001 & n.a. & 0,07 & n.a. & n.a. & n.a. & n.a. & 770 \\
\hline 2beng & 0,33 & n.a. & 0,24 & n.a. & 0,24 & n.a. & 0,003 & n.a. & 0,001 & n.a. & 0,09 & 0,970 & 460,00 & 440,00 & 670 \\
\hline 3eng & 0,18 & 0,19 & 0,14 & 0,02 & 0,10 & 0,003 & 0,002 & 0,003 & 0,001 & 0,26 & 0,28 & 0,818 & 440,00 & 420,00 & 650 \\
\hline 5eng & 0,26 & 0,34 & 0,33 & 0,05 & 0,18 & 0,015 & 0,002 & 0,004 & 0,001 & 0,28 & 0,26 & 0,985 & 360,00 & 320,00 & 1340 \\
\hline 8eng & 0,57 & 1,35 & 1,08 & 0,15 & 0,16 & 0,005 & 0,002 & 0,001 & 0,001 & 0,53 & 0,53 & 0,924 & 740,00 & 720,00 & 340 \\
\hline 16eng & 0,15 & 0,16 & 0,17 & 0,02 & 0,16 & 0,003 & 0,002 & 0,001 & 0,001 & 0,28 & 0,31 & 0,811 & 220,00 & 160,00 & 450 \\
\hline 18 eng & 0,20 & 0,33 & 0,33 & 0,04 & 0,16 & 0,004 & 0,002 & 0,001 & 0,001 & 0,27 & 0,30 & 1,098 & 300,00 & 280,00 & 390 \\
\hline 19eng & 0,21 & 0,11 & 0,13 & 0,05 & 0,20 & 0,005 & 0,003 & 0,002 & 0,001 & 0,18 & 0,22 & 0,894 & 200,00 & 200,00 & 230 \\
\hline $22 \mathrm{eng}$ & 0,10 & 0,05 & 0,03 & 0,02 & 0,23 & 0,004 & 0,003 & 0,006 & 0,001 & 0,07 & 0,10 & 0,902 & 180,00 & 180,00 & 1170 \\
\hline $24 \mathrm{eng}$ & 0,08 & 0,23 & 0,27 & 0,31 & 0,42 & 0,006 & 0,006 & 0,007 & 0,001 & 0,37 & 0,38 & 0,939 & 700,00 & 660,00 & 4730 \\
\hline 26eng & 5,17 & 6,92 & 7,63 & 0,00 & 0,19 & 0,003 & 0,003 & 0,000 & 0,001 & 3,11 & 3,58 & 0,652 & 3140,00 & 3120,00 & 320 \\
\hline 27 eng & 0,08 & 0,05 & 0,09 & 0,01 & 0,16 & 0,004 & 0,002 & 0,000 & 0,001 & 0,20 & 0,24 & 0,917 & 220,00 & 180,00 & 1160 \\
\hline $28 \mathrm{eng}$ & 0,78 & 1,57 & 1,38 & 0,03 & 0,18 & 0,003 & 0,002 & 0,000 & 0,001 & 1,48 & 1,05 & 0,697 & 720,00 & 660,00 & 1870 \\
\hline $29 \mathrm{eng}$ & 1,31 & 1,14 & 2,30 & 0,00 & 0,14 & 0,008 & 0,002 & 0,002 & 0,001 & 0,90 & 1,20 & 0,886 & 1200,00 & 1120,00 & 3500 \\
\hline 30eng & 2,83 & 10,67 & 9,90 & 0,18 & 0,15 & 0,005 & 0,002 & 0,001 & 0,001 & 1,63 & 1,57 & 0,583 & 2380,00 & 2320,00 & 620 \\
\hline $\begin{array}{l}\text { Ichnusa } \\
\text { well } 1\end{array}$ & 0,18 & n.a. & 0,15 & n.a. & 0,21 & n.a. & 0,003 & n.a. & 0,002 & n.a. & 0,16 & 0,977 & 420,00 & 320,00 & 600 \\
\hline
\end{tabular}




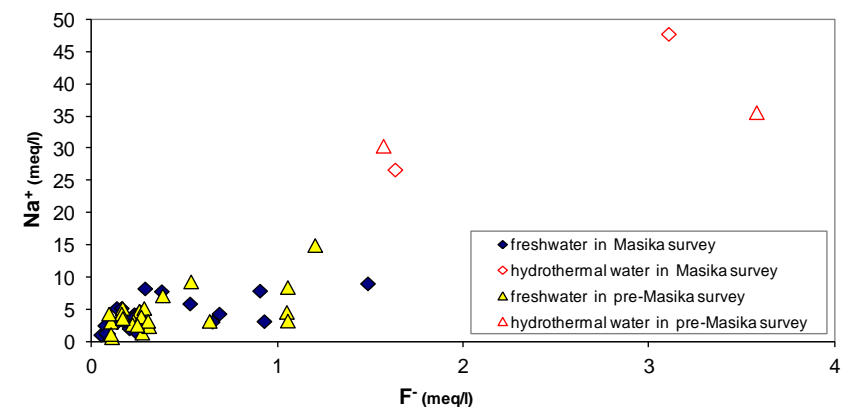

Fig. 4. Sodium vs. fluoride scatter diagram of all groundwater samples.

diffracted beam. The following operating conditions were applied: $40 \mathrm{kV}, 30 \mathrm{~mA}, 2 \theta$ range $2-70^{\circ}$, step size $0.02^{\circ}, 2 \mathrm{~s}$ step $^{-1}$. Wet milling was avoided due to the possible presence of highly soluble phases; instead powders were prepared by hand in an agate mortar. Minerals were identified using Bruker EVA v14.2 software (Bruker AXS, 2008) and the PDF-2 database (ICDD, 2003). All statistical analyses were based on R v2.7.0 software (http://www.r-project.org/).

\subsection{Results}

\subsubsection{Chemistry}

The physico-chemical properties of the groundwater samples varied markedly (Table 1 ). The groundwater $\mathrm{pH}$ varies from 5.9 to 8.1. The Electrical conductivity (EC) varies from 190 to $5070 \mu \mathrm{Scm}^{-1}$. Sodium and $\mathrm{HCO}_{3}^{-}$are the dominant ions ranging, from 24.5 to $1100 \mathrm{mg}^{-1}$ and from 89.5 to $2143 \mathrm{mg} \mathrm{l}^{-1}$ respectively. The concentration of fluoride in the groundwater varies from 0.90 to $68.00 \mathrm{mg} \mathrm{l}^{-1}$. In the most of groundwater samples $(84 \%), \mathrm{F}^{-}$exceeds the WHO limit $\left(1.5 \mathrm{mg}^{-1}\right)$, whereas the $70 \%$ are above the Tanzanian limit $\left(4 \mathrm{mg}^{-1}\right)$. These, mainly, spring up from lahar formations (Fig. 2).

The ternary diagram analysis identified a unique water type, namely bicarbonate-alkaline-earth (Fig. 3).

Chloride concentrations were relatively low, as is the case for most natural water systems (Davies, 1996), while the concentration of the other major anions was rather variable. As we will show later on, the presence of bicarbonate and sodium ions (Figs. 4 and 5) suggested that in bicarbonate-alkaline-earth waters, the presence of bicarbonate and sodium ions may exert an influence over the fluoride concentration.

The river waters also shows a bicarbonate-alkaline hydrofacies, in particular at the three collection sites along the Ngarenanyuki river (river samples 1, 24 and 28), where the concentration of major ions and fluoride was higher than in the other river water samples (Fig. 6).

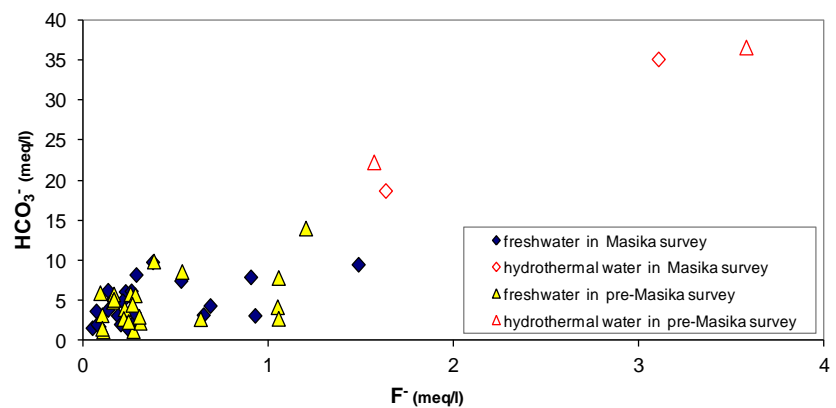

Fig. 5. Bicarbonate vs. fluoride scatter diagram of all groundwater samples.
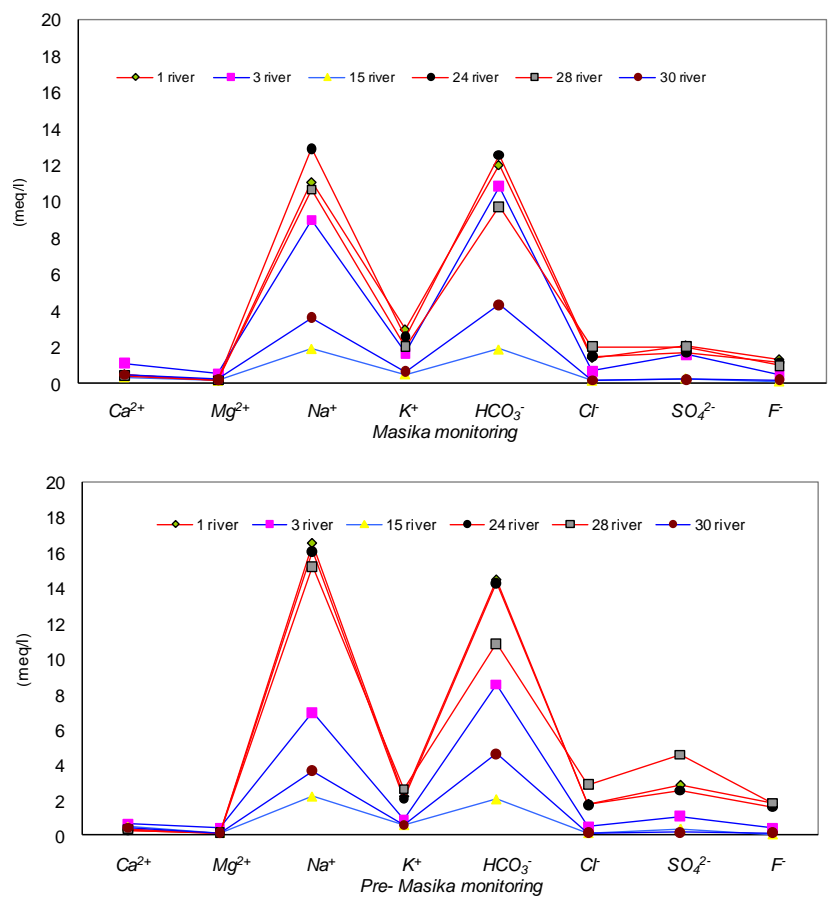

Fig. 6. Distribution of the major ions in river water samples.

\subsubsection{Mineralogy}

The outcome of the X-ray analysis applied to the sample subset is given in Table 2. The more (ENG6; OLD11) or less (OLD9, ENG1; ENG31) pronounced Gaussian shape of the background in the $\approx 15-40^{\circ} 2 \theta$ region showed that, in addition to the crystalline phases, a glassy fraction was also present in all samples. The phonolite rock samples (ENG1 and ENG31) contain alkali feldspar and feldspathoids, along with riebeckite, augite, fluroapatite; these phases also occur, with only minor exceptions, in all samples. Among the authigenic minerals, the zeolites phillipsite and chabazite (which are ubiquitous in lake deposits and frequently found in lahars) were formed because of the interaction between volcanic glass and alkaline solutions; cancrinite (limited to 
Table 2. Mineral composition of sediments and rocks samples.

\begin{tabular}{|c|c|c|c|c|c|c|c|c|c|c|c|c|}
\hline & $\begin{array}{l}\text { Paleo- } \\
\text { soil } \\
\text { Lahar } \\
(\mathrm{Nzd} 2)\end{array}$ & $\begin{array}{l}\text { Lahar } \\
(\mathrm{Nzd} 2)\end{array}$ & $\begin{array}{l}\text { Lake } \\
\text { deposits } \\
\text { (1) }\end{array}$ & $\begin{array}{l}\text { Crusts } \\
\text { of } \\
\text { Lake } \\
\text { deposits } \\
\text { (1) }\end{array}$ & $\begin{array}{l}\text { Lake } \\
\text { deposits } \\
\text { (1) }\end{array}$ & $\begin{array}{l}\text { Crusts } \\
\text { of } \\
\text { Lake } \\
\text { deposits } \\
\text { (1) }\end{array}$ & $\begin{array}{l}\text { Breccias } \\
\text { on } \\
\text { Mantling } \\
\text { Ash } \\
\text { (Nvf) }\end{array}$ & $\begin{array}{l}\text { Lahar } \\
\text { (Nzd1) }\end{array}$ & $\begin{array}{l}\text { Phonolite } \\
(\mathrm{Nvm})\end{array}$ & $\begin{array}{l}\text { Calcrete } \\
\text { on } \\
\text { Mantling } \\
\text { Ash } \\
\text { (Nvf) }\end{array}$ & $\begin{array}{l}\text { Silt } \\
\text { Layer } \\
\text { in } \\
\text { soil }\end{array}$ & $\begin{array}{l}\text { Phonolite } \\
\text { (Nvm) }\end{array}$ \\
\hline & ENG6 & ENG7 & ENG8 & ENG8b & $2474 \mathrm{~S}$ & $2474 \mathrm{C}$ & OLD $3,4,5$ & OLD 10 & ENG1 & OLD9 & OLD11 & ENG31 \\
\hline Phillipsite & & $\mathrm{x}$ & $\mathrm{x}$ & $\mathrm{x}$ & $\mathrm{x}$ & $\mathrm{x}$ & & $\mathrm{x}$ & & & & \\
\hline Chabazite & & $\mathrm{x}$ & $\mathrm{x}$ & $\mathrm{x}$ & $\mathrm{x}$ & $\mathrm{x}$ & & $\mathrm{x}$ & & & & \\
\hline Analcime & & $\mathrm{x}$ & $\mathrm{x}$ & $\mathrm{x}$ & $\mathrm{x}$ & $\mathrm{x}$ & & & $\mathrm{x}$ & & & \\
\hline Nepheline & $\mathrm{x}$ & $\mathrm{x}$ & $\mathrm{x}$ & $\mathrm{x}$ & $\mathrm{x}$ & $\mathrm{x}$ & $\mathrm{x}$ & $\mathrm{x}$ & $\mathrm{x}$ & & $\mathrm{x}$ & $\mathrm{x}$ \\
\hline Leucite & $\mathrm{x}$ & $\mathrm{x}$ & $\mathrm{x}$ & $\mathrm{x}$ & $\mathrm{x}$ & $\mathrm{x}$ & & & $\operatorname{tr}$ & & & $\operatorname{tr}$ \\
\hline Anorthoclase & $\mathrm{x}$ & $\mathrm{x}$ & $\mathrm{x}$ & $\mathrm{x}$ & $\mathrm{x}$ & $\mathrm{x}$ & $\mathrm{x}$ & $\mathrm{x}$ & $\mathrm{x}$ & $\mathrm{x}$ & $\mathrm{x}$ & $\mathrm{x}$ \\
\hline Sanidine & $\mathrm{x}$ & $\mathrm{x}$ & $\mathrm{x}$ & & $\mathrm{x}$ & & $\mathrm{x}$ & $\mathrm{x}$ & $\mathrm{x}$ & $\mathrm{x}$ & $\mathrm{x}$ & $\mathrm{x}$ \\
\hline Albite & & $\mathrm{x}$ & $\mathrm{x}$ & & & & & & & & $\mathrm{x}$ & \\
\hline Riebeckite & & $\mathrm{x}$ & $\mathrm{x}$ & & & $\mathrm{x}$ & & $\mathrm{x}$ & & & $\mathrm{x}$ & $\mathrm{x}$ \\
\hline Augite & $\mathrm{x}$ & $\mathrm{x}$ & $\mathrm{x}$ & $x$ & $\mathrm{x}$ & $\mathrm{x}$ & $\mathrm{x}$ & $\mathrm{x}$ & $\mathrm{x}$ & & $\mathrm{x}$ & $\mathrm{x}$ \\
\hline Biotite & & $\mathrm{x}$ & $\mathrm{x}$ & $\mathrm{x}$ & & $\mathrm{x}$ & $\mathrm{x}$ & & & & & \\
\hline Illite/smectite & $\mathrm{x}$ & & & & & & & & & $\mathrm{x}$ & $\mathrm{x}$ & \\
\hline Trona & & & & $\mathrm{x}$ & & $\mathrm{x}$ & & & & & & \\
\hline Natron & & & $\operatorname{tr}$ & & & & & & & & & \\
\hline Natrite & $\mathrm{x}$ & & $\operatorname{tr}$ & $\mathrm{x}$ & & & & & $\mathrm{x}$ & & & \\
\hline Calcite & & & & & & $\mathrm{x}$ & $\mathrm{x}$ & & & $\mathrm{x}$ & & \\
\hline Cancrinite & & $\mathrm{x}$ & $\mathrm{x}$ & & & $\operatorname{tr}$ & & $\mathrm{x}$ & & & & \\
\hline Sylvite & & & $\mathrm{x}$ & $\mathrm{x}$ & $\mathrm{x}$ & & & & & & & \\
\hline Fluorapatite & & $\mathrm{x}$ & $\mathrm{x}$ & & $\mathrm{x}$ & $\mathrm{x}$ & & $\mathrm{x}$ & $\mathrm{x}$ & & & $\mathrm{x}$ \\
\hline Aphthitalite & & & & $\mathrm{x}$ & & & & & & & & \\
\hline Goethite & & & & $\mathrm{x}$ & & & & & & & & \\
\hline
\end{tabular}

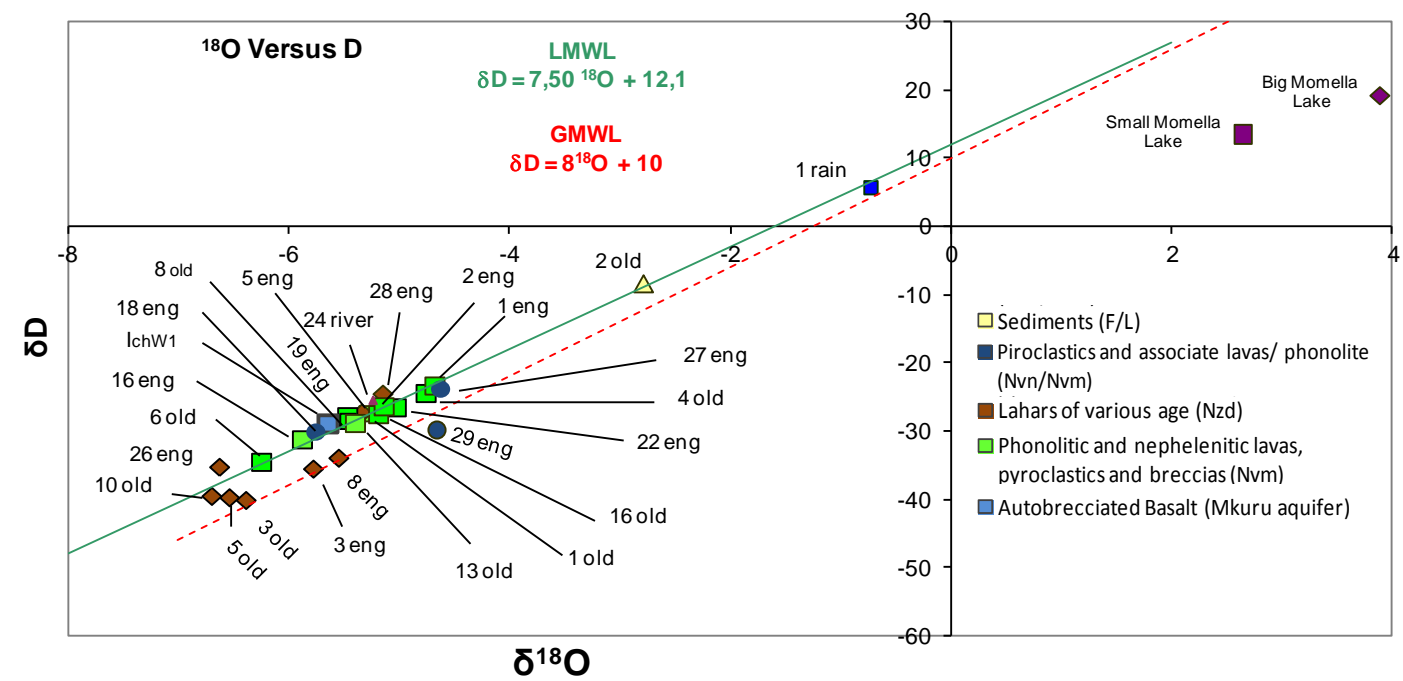

Fig. 7. Plot of ${ }^{2} \mathrm{H}$ vs. ${ }^{18} \mathrm{O}$ content. The Local Meteoric Water Line (LMWL) was defined following Dettman et al. (2005), whereas the Global Meteoric Water Line (GMWL) by Craig (1961). 
Table 3. Isotopic water analysis and composition.

\begin{tabular}{|c|c|c|c|c|c|}
\hline \multirow{2}{*}{$\begin{array}{l}\text { ID } \\
\text { sample }\end{array}$} & \multirow[b]{2}{*}{ Date } & \multirow{2}{*}{$\begin{array}{r}\delta^{18} \mathrm{O} \% \text { o } \\
\text { V-SMOW }\end{array}$} & \multirow{2}{*}{$\begin{array}{r}\delta \mathrm{D} \\
\mathrm{V}-\mathrm{SMOW}\end{array}$} & \multicolumn{2}{|c|}{${ }^{3} \mathrm{H}$} \\
\hline & & & & (U.T.) & \pm (U.T.) \\
\hline 1old & 19 February 2007 & $-5,3$ & -28 & 1,2 & 0,6 \\
\hline 2old & 21 February 2007 & $-2,8$ & -8 & 2,6 & 0,7 \\
\hline 3old & 21 February 2007 & $-6,4$ & -40 & 1,6 & 0,6 \\
\hline 4old & 22 February 2007 & $-4,8$ & -24 & 1,7 & 0,6 \\
\hline 5old & 22 February 2007 & -6.54 & -39 & 0.9 & 0.6 \\
\hline 6old & 17 April 2007 & $-6,3$ & -35 & 0.4 & 0.3 \\
\hline 8old & 17 April 2007 & -5.47 & -29 & 1,1 & 0,4 \\
\hline 10old & 17 April 2007 & -6.70 & -40 & 0.8 & 0.3 \\
\hline 13old & 16 April 2007 & $-5,4$ & -29 & 1,1 & 0,4 \\
\hline 16old & 16 April 2007 & $-5,2$ & -28 & 2,2 & 0,5 \\
\hline 1eng & 18 February 2007 & $-4,7$ & -23 & 1,1 & 0,6 \\
\hline 2eng & 18 February 2007 & $-5,1$ & 17 & n.a. & \\
\hline 3eng & 21 February 2007 & $-5,8$ & -36 & 1,1 & 0,6 \\
\hline 5eng & 22 April 2007 & $-5,3$ & -27 & 1,0 & 0,4 \\
\hline 8eng & 17 February 2007 & $-5,6$ & -34 & 0,8 & 0,6 \\
\hline 16eng & 22 April 2007 & $-5,9$ & -31 & 0,7 & 0,4 \\
\hline 18 eng & 18 April 2007 & $-5,8$ & -30 & 0,8 & 0,3 \\
\hline $19 \mathrm{eng}$ & 18 April 2007 & $-5,5$ & -28 & 1,0 & 0,4 \\
\hline $22 \mathrm{eng}$ & 20 February 2007 & $-5,0$ & -27 & 2,4 & 0,7 \\
\hline $26 \mathrm{eng}$ & 05 April 2007 & $-6,6$ & -35 & 1,5 & 0,5 \\
\hline 27 eng & 05 April 2007 & $-4,6$ & -24 & 2,2 & 0,5 \\
\hline 28eng & 05 April 2007 & $-5,2$ & -25 & 1,7 & 0,5 \\
\hline 29eng & 05 April 2007 & $-4,7$ & -30 & 2,2 & 0,5 \\
\hline Ich. well 1 & 18 January 2008 & $-5,6$ & -29 & 1,5 & 0,6 \\
\hline 24 river & 23 April 2007 & $-5,2$ & -25 & 1,5 & 0,4 \\
\hline Big M.L. & 17 February 2007 & 3,9 & 19 & 1,8 & 0,7 \\
\hline Small M.L. & 17 February 2007 & 2,7 & 14 & 2,1 & 0,7 \\
\hline Rain & 22 April 2007 & $-0,7$ & 6 & 2,8 & 0,9 \\
\hline
\end{tabular}

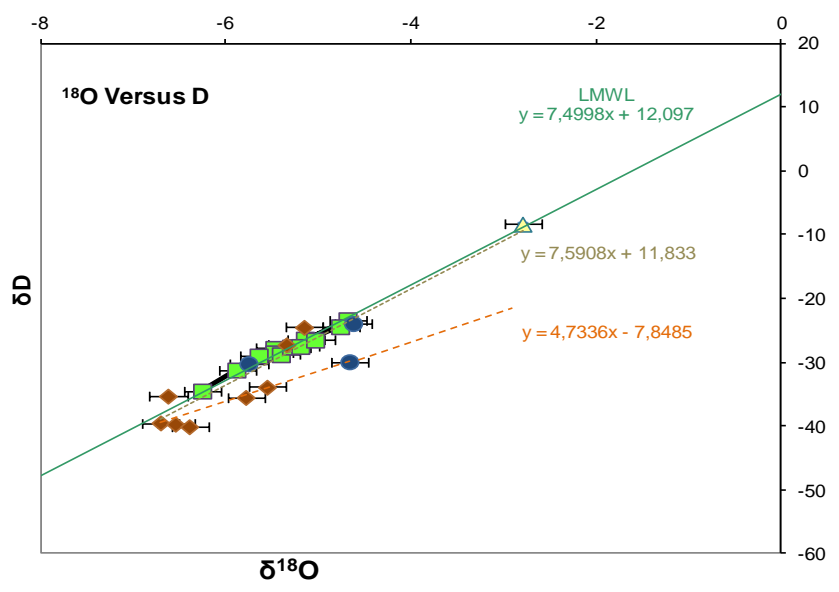

Fig. 8. Plot of ${ }^{2} \mathrm{H}$ vs. ${ }^{18} \mathrm{O}$ content with groundwater and evaporation regression line. lahars and lake deposits) can be considered as a newlyformed phase, while analcime can be a magmatic as well as a secondary-formed phase. Nepheline and leucite are magmaticin this context, and represent detrital minerals in sedimentary lithotypes. Clay minerals, belonging to the illite/smectite group, were found in paleosoils interbedded within various lahar (ENG6, OLD9 and OLD11). Trona, natron, natrite, sylvite, aphthitalite and calcite were present, though never all together. These phases can be directly precipitated from super-saturated solutions and frequently form, sometimes in conjunction with zeolites, crusts, hardpans and calcrete, as in the calcrete OLD9, in breccias (OLD3, 4 and 5) and in the crust of a lake deposit (2474 C). Trona is referred to locally as "scooped magadi" when it forms an efflorescent crust on the soil surface in association with mixtures of halite, quartz, villiaumite, kogarkoite and thermonatrite (Nielsen, 1999). This assemblage was not present in samples in which trona was associated with either natrite, sylvite, aphthitalite, goethite, phillipsite, chabazite and analcime of probably secondary formation (ENG8b), or 


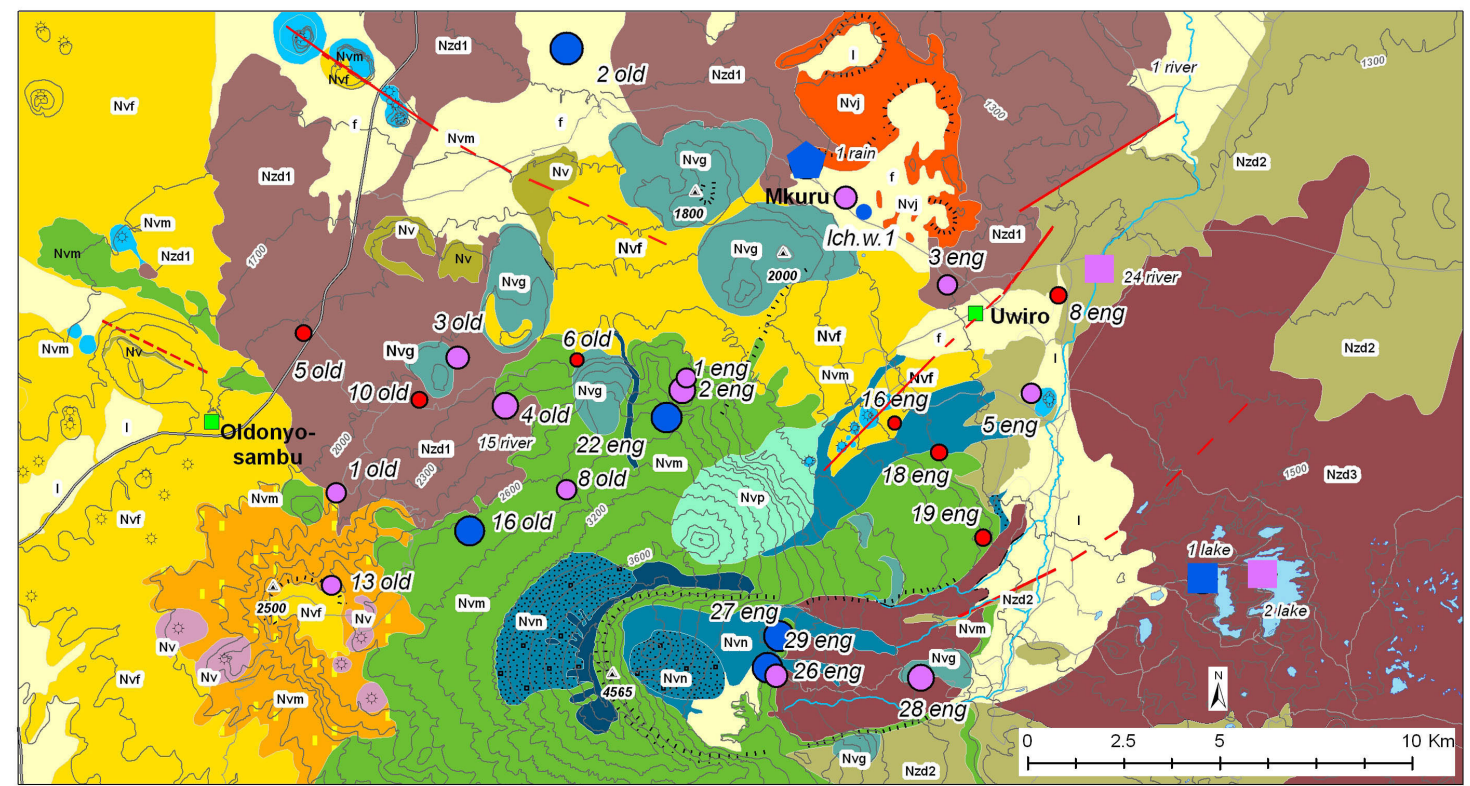

SEDIMENTARY HYDROGEOLOGICAL UNIT

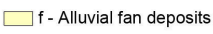

$\square$ I- Lake deposits
VOLCANIC HYDROGEOLOGICAL UNIT

$$
\begin{aligned}
& \text { Nvn - Piroclastics (stippled) and associate lavas - } \\
& \text { [Ash cone group complex] } \\
& \text { Nzd3 - Momella lahars - [Lahars of various age complex] } \\
& \text { Nzd2 - Ngare Nanyuki lahars - [Lahars of various age } \\
& \text { complex] } \\
& \text { Nvf - Mantling ash - [Mantling Ash complex] } \\
& \text { Nvn - Nephelinitic and phonolitic lavas (Summit group) - } \\
& \text { [Main Cone group complex] } \\
& \text { Nvm - Pyroclastics with subordinate nephelenitic and } \\
& \text { phonolitic lavas - [Main Cone group complex] } \\
& \text { Nvg - Tholoids - [Main Cone group complex] }
\end{aligned}
$$

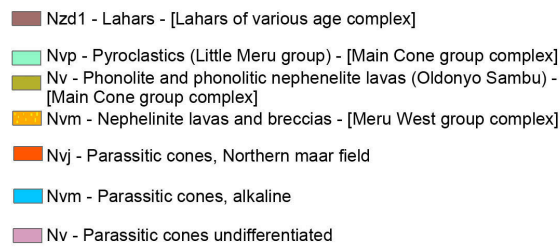

$\square$ Nv - Parassitic cones undifferentiated

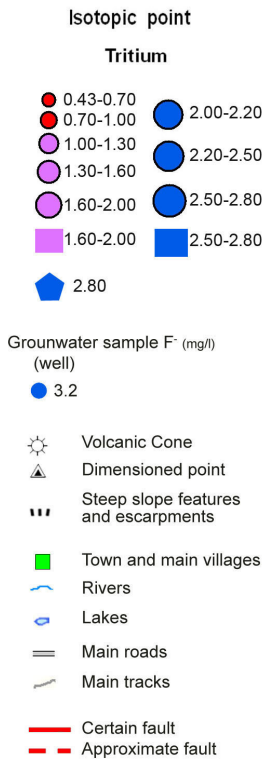

Fig. 9. Distribution map of the study area, indicating the location of isotopic water points classified according to their ${ }^{3} \mathrm{H}$ content.

calcite, cancrinite, phillipsite, chabazite and secondary analcime $(2474 \mathrm{C})$. The respective underlying samples (ENG8 and $2474 \mathrm{~S}$, just below the crust) were richer in primary minerals and contained neither trona, calcite nor aphthitalite. Only traces of natron and natrite were identified in ENG8.

\subsubsection{Isotopic data}

Plot of Fig. 7 shows two major water groups: the first, containing the majority of samples, falls on the local meteoric water line (LMWL) and the second below this line.

The equation related to the first group gives a line (Fig. 8) that basically overlaps that reported by Dettman et al. (2005), despite the limited number of samples collected only in the masika period.

However, the sample derived from local recharge (2OLD) differ from groundwater with deep circulation which infiltrate in the Mt. Meru highs; clearly those waters reflect a depletion in $\delta \mathrm{D}$ and $\delta^{18} \mathrm{O}$. The most depleted samples (10 OLD, $6 \mathrm{OLD}, 26 \mathrm{ENG}$ ) also provide the lower values in ${ }^{3} \mathrm{H}$ (Fig. 9).

The second group which shifts below the LMWL (3 OLD, 5 OLD, 3 ENG, 8 ENG, 29 ENG) gives a regression line with different slope (Fig. 8) . Such a slope ranging between 36, according to Craig (1961) and Clark and Fritz (1997), is representative of a non-equilibrium evaporation responsible for higher enrichment of ${ }^{18} \mathrm{O}$ with respect of $\mathrm{D}$.

As reported in Fig. 8 these waters, probably came from an original water (10 OLD), that recharge at great altitude and spring up in the Oldonyo Sambu slope, shows a more negative isotopic value of $\delta^{18} \mathrm{O}\left(-6.7 \%\right.$ ) and rather low ${ }^{3} \mathrm{H}$ content suggesting a long residence time (Fig. 9).

In general, on the slope and in the lowland area, the waters were characterized by a rather low ${ }^{3} \mathrm{H}$ content. At higher altitudes, with only few exceptions, the water had a high ${ }^{3} \mathrm{H}$ content (Fig. 9). Here, the groundwater was likely to have been largely recharged via the infiltration of rainwater through fractures, in line with hydrogeologic evidence (Ghiglieri et al., 2010).

\subsubsection{Fluoride distribution}

The distribution of fluoride was determined by a combination of altitude and aquifer lithology. Within phonolite and basalt aquifers, the fluoride level was lowest on the phonolite outcrops at higher altitudes $\left(1.6 \mathrm{mg}^{-1}\right)$, whereas the concentration in water emerging from basalt at the base of the Mt. Meru cone reached $7.2 \mathrm{mg}^{-1}$. 


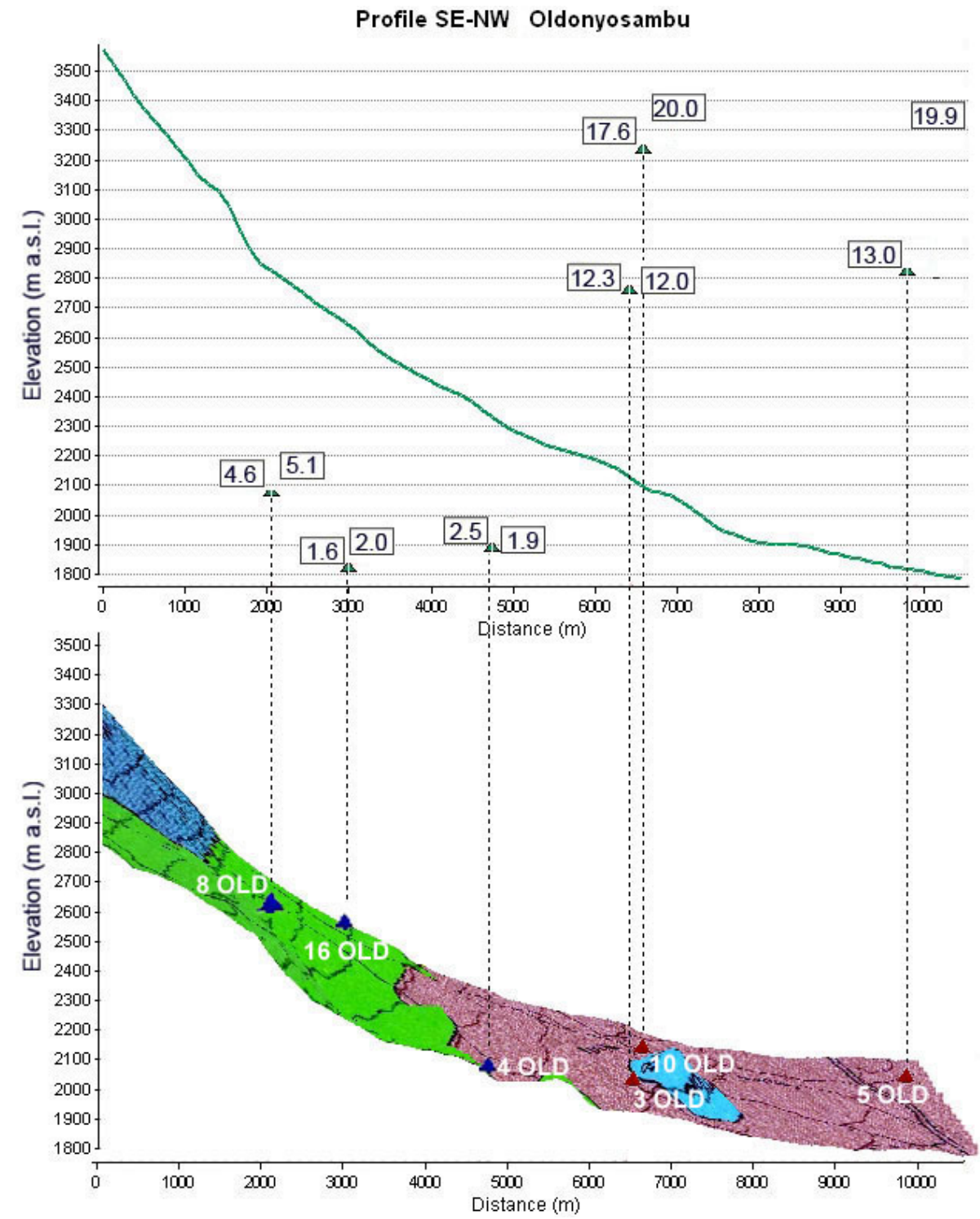

Fig. 10. Cross section of the NW side of Mt. Meru, showing the location of the springs and the fluoride content of the spring waters (mg $\mathrm{l}^{-1}$ ) for the Masika (left) and pre-Masika (right) survey.

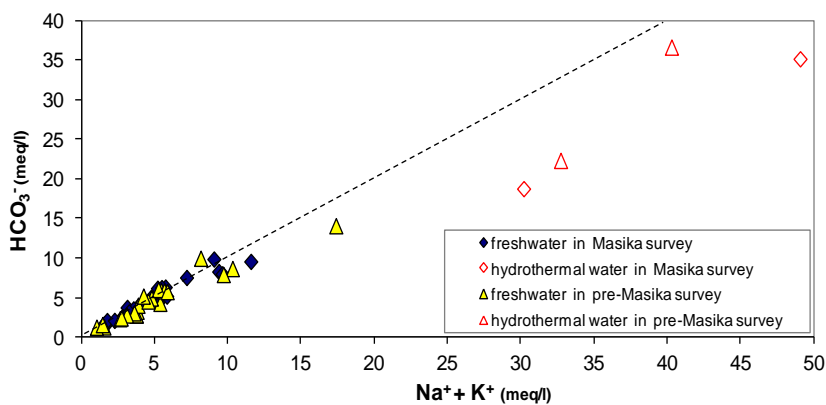

Fig. 11. Plot of $\mathrm{Na}^{+}+\mathrm{K}^{+}$vs. alkalinity.

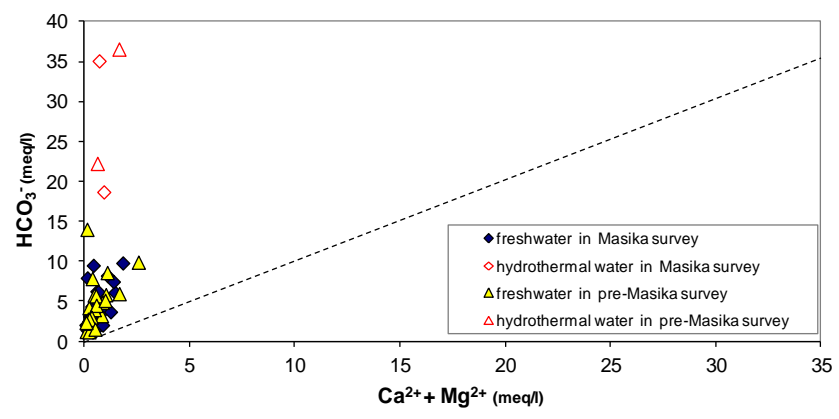

Fig. 12. Plot of $\mathrm{Ca}^{2+}+\mathrm{Mg}^{2+}$ vs. alkalinity. 


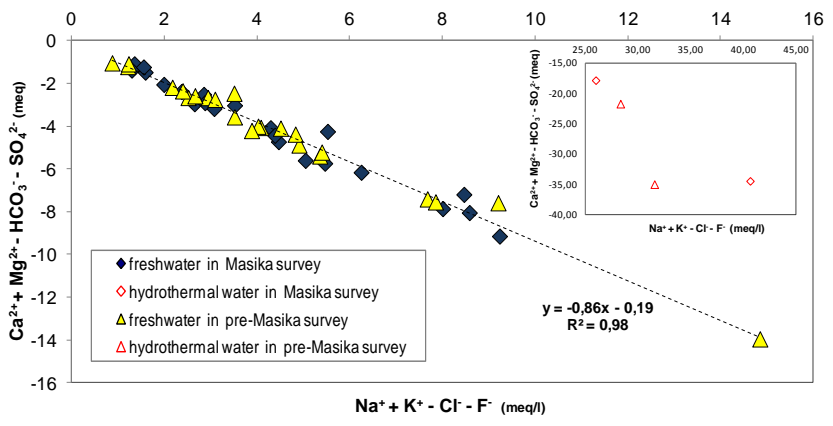

Fig. 13. A Ca-Mg-alkalinity-SO 4 vs. Na-K-Cl-F scatter diagram illustrates the occurrence of ion exchange. The dotted line represents the $1: 1$ ratio.

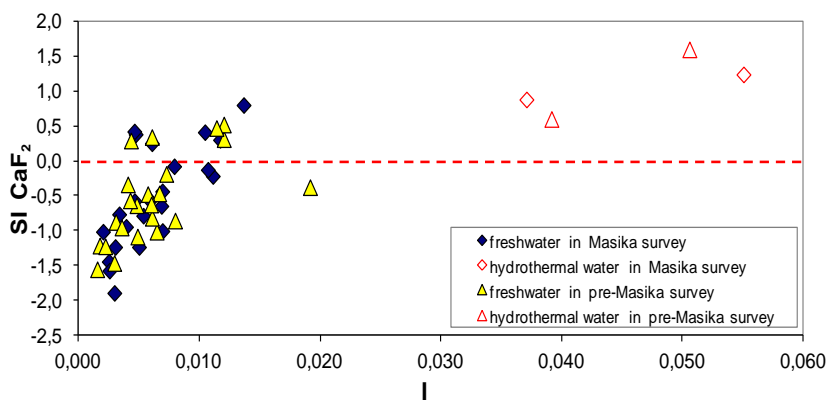

Fig. 14. Plot of fluorite saturation index vs. ionic strength.

The highest contents were in the water emerging from lahar hosted aquifers in the flat land within the Uwiro graben. Figure 10 shows the flow route from the recharge zone of the NW side Mt. Meru (phonolite) to the Oldonyo Sambu valley (lahar), and documents the fluoride content of a number of local springs. The $26 \mathrm{ENG}$ spring (along with $28 \mathrm{ENG}$ and $29 \mathrm{ENG}$ ) were the exceptions to the trend of fluoride content decreasing with altitude. Its water had a constant temperature of $22.4^{\circ} \mathrm{C}$ and a depleted ${ }^{3} \mathrm{H}$ content, which together suggested that the spring is of hydrothermal origin; this would account for its showing the highest fluoride content so far detected in the study area. 26 ENG feeds the Engarenanyuky river at a rate of $61 \mathrm{~s}^{-1}$, and thus represents a source of pollution for both the river and any aquifers hydraulically connected it, such as in the area lying on the Uwiro graben lowland.

\section{Insights gained from the physico-chemical analysis}

\subsection{The dissolution of major ions}

Typically, silicate minerals and glass present in alkaline lava and ash are weathered by hydrolysis, producing $\mathrm{NaHCO}_{3}$ rich and $\mathrm{Ca}^{2+}$ and $\mathrm{Mg}^{2+}$ depleted groundwater (Jones et al., 1977). Here, this process affected the degradation

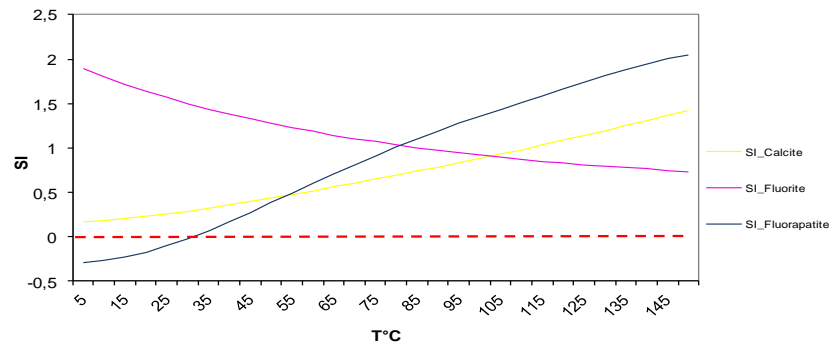

Fig. 15. Plot of the saturation indices of several mineral phases vs. temperature $\left({ }^{\circ} \mathrm{C}\right)$ for the water of spring $26 \mathrm{ENG}$.

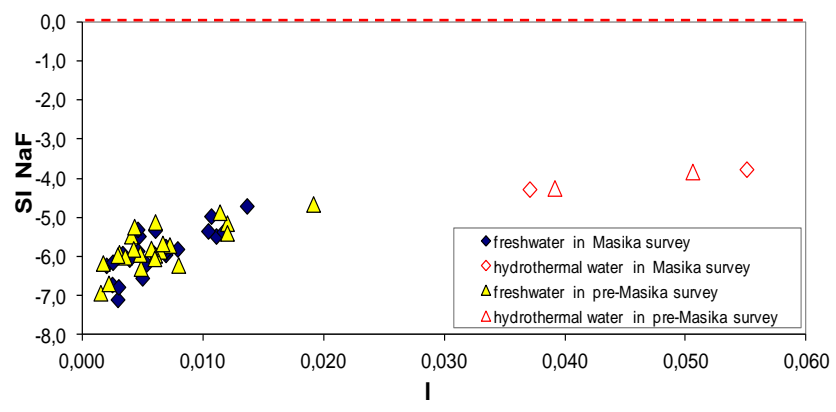

Fig. 16. Plot of villiaumite saturation index vs. ionic strength.

of phonolite, tephrite-phonolite, Na-K-feldspar and Na-Kfeldspatoid, which occurred in the lahar formations. Groundwaters, particularly those containing dissolved $\mathrm{CO}_{2}$, react readily with alkaline silicate such as albite to release sodium and bicarbonate ions. This process appears to be commonplace in the NE-SW-trending fault system on the eastern flank of Mt. Meru, where the most suitable hydrothermal conditions occur. The alkalinity of groundwater is, therefore, strictly associated with the presence of alkali metals (Jalali, 2007), as demonstrated by the positive correlation between alkalinity (bicarbonate content) and $\left(\mathrm{Na}^{+}+\mathrm{K}^{+}\right)$of Fig. 11. On the contrary (Fig. 12) no correlation was observed between alkalinity and alkali earth metals $\left(\mathrm{Ca}^{2+}+\mathrm{Mg}^{2+}\right)$.

\subsection{Exchange processes of the major ions}

The exchange capacity process affects secondary mineral associations such as zeolite and other sodium-hydrate silicates characteristically formed in an high evaporation environment (Sebag et al., 2001). These minerals are able not only to concentrate cations, but also to release them in response to changes in water availability and temperature which occur as a result of normal seasonal cycling.

By influencing the concentration of cations in the water, the zeolites can indirectly play a role on fluorine distribution, permitting or preventing the precipitation of F-bearing phases, namely fluorides. As shown in Fig. 13, there is a 


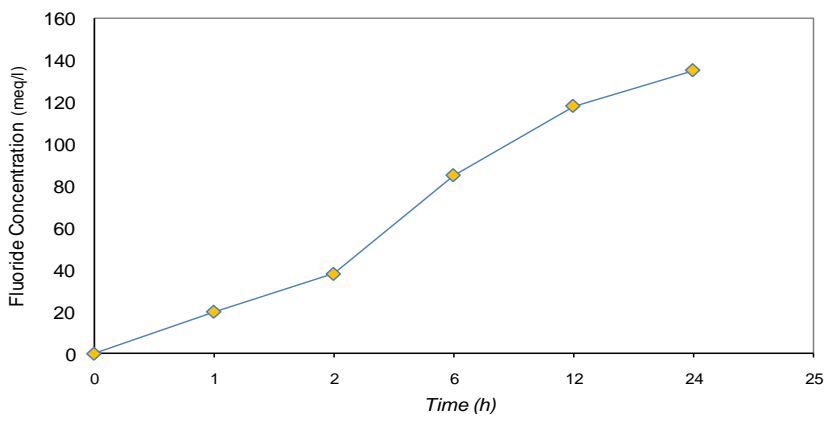

Fig. 17. A leaching test of a mantling ash sample conducted at ambient temperature.

marked negative correlation between $\mathrm{Na}^{+}+\mathrm{K}^{+}-\mathrm{Cl}^{-}-\mathrm{F}^{-}$and $\mathrm{Ca}^{2+} \mathrm{Mg}^{2+}-\mathrm{HCO}_{3}^{-}-\mathrm{SO}_{4}^{2-}$. According to $\mathrm{Su}$ et al. (2007), samples lying furthest from the origin have experienced the greatest extent of ionic exchange.

\subsection{Fluoride content}

Since the presence/absence of certain minerals shows which minerals have been dissolved/ precipitated, saturation index calculations were derived for each of the groundwater samples. The fluoride content of the groundwaters was verified by the positive relationship existing between the saturation index (SI) and the ionic strength $(I)$. When $\mathrm{SI}<1$, minerals tend to dissolve, while at SI $>1$, they tend to be precipitated. The SI values for fluorite $(-1.89-+1.25$ in the Masika samples, and 1.55-1.61 in the pre-Masika samples) and those for $I(0.002-0.05)$ suggested that the phonolite and basalt groundwaters were sub-saturated with respect to fluorite, while those emerging from lahar and sedimentary rocks were at equilibrium between the mineral phase and the solution (Fig. 14). The data can also be used to infer the residence time of the groundwater, and these inferences largely supported the ${ }^{3} \mathrm{H}$-derived conclusions, such that residence time of the groundwater in the phonolite formations was short, while that in the lahars was somewhat longer.

Although these data may be predictive with respect to fluorite super-saturation, this phase was not identified in the study area, presumably because of the scarcity of dissolved calcium as scavenged by $\mathrm{CaCO}_{3}$ precipitation according to:

$\mathrm{CaF}_{2}+2 \mathrm{HCO}_{3}^{-}=\mathrm{CaCO}_{3}+2 \mathrm{~F}^{-}+\mathrm{H}_{2} \mathrm{O}+\mathrm{CO}_{2}$

Calcrete and hardpan were noted at the base of these soil profiles, and travertine concretion on the porous lava flows. Calcium removal by the precipitation of calcite allows the fluoride concentration to increase (Kim and Young Jeong, 2005).

Ionic exchange can also enhance calcium capture and sodium release. The variation in the 26 ENG SI with respect to various minerals at various temperatures is shown in Fig. 15. Sub-saturation values for fluorite were not reached at temperatures $>150^{\circ} \mathrm{C}$. At the temperature of the emerging spring water $\left(22^{\circ} \mathrm{C}\right)$, this phase is insoluble. Thus it may be that fluorapatite was the source of fluoride in the water.

$$
\begin{aligned}
& \mathrm{Ca}_{5}\left(\mathrm{PO}_{4}\right)_{3} \mathrm{~F}+6 \mathrm{CO}_{2}+6 \mathrm{H}_{2} \mathrm{O} \rightarrow 5 \mathrm{Ca}^{2+} \\
& +3 \mathrm{H}_{2} \mathrm{PO}_{4}^{-}+\mathrm{F}^{-}+6 \mathrm{HCO}_{3}^{-}
\end{aligned}
$$

As shown in the plot its behaviour is antithetic with respect to the $\mathrm{CaF}_{2}$. At a temperature $>36^{\circ} \mathrm{C}$, fluorapatite precipitates, so the expectation is that this mineral would remain sub-saturated in the groundwater. In supergene condition, a further source of fluoride can be provided by secondary mineral associations (Nielsen, 1999; Vuhahulaa et al., 2008), which are favoured by the high level of evaporation typical of this climatic region (Lahermo et al., 1991).

An increased $\mathrm{Na}^{+} / \mathrm{Ca}^{2+}$ ratio could theoretically favour the fractionation of fluoride by villiaumite, a mineral phase which is extremely soluble (Gao et al., 2007). However, the groundwaters were uniformly strongly sub-saturated with respect to villiaumite (Fig. 14). Even though villiaumite may be present in evaporative lakes (Kilham and Hecky, 1973), it was not detected here either in saline crusts or in association with scooped magadi. A ready source of alkali and fluoride (up to $3700 \mathrm{ppm}$ ) is also presented by the scooped magadi and crusts of the sediment cover. Thus, the fluoride rich Ngarenanyuki river water, in combination with leachate from the scooped magadi, may well have contributed to the increased fluoride content in the lahar aquifer.

A further important source of fluoride is represented by the ubiquitous volcanic ash (not present on the higher slopes of Mt. Meru). A leaching test conducted at ambient temperature on a representative sample of slightly altered, yellowish, powder-like ash showed that a considerable amount of fluoride can be readily released (Fig. 15), explaining the high fluoride content of alkaline magmas. The melting of Na-phonolite shows a higher diffusivity of fluoride (BalconeBoissard et al., 2009) with respect chloride, and as opposed to $\mathrm{H}_{2} \mathrm{O}$ and $\mathrm{S}$ hardly degass from the melt so giving rise to fluoride-rich glassy ejecta (Signorelli et al., 1999).

\section{Conclusions}

The chemistry of the groundwaters showed that a variety of natural processes must have been responsible both for the diversity of the hydrochemical facies and for the presence of fluoride contamination. Chief among these processes are dissolution, exchange capacity and precipitation from supersaturated solutions. A fluoride contamination model demonstrated that an interaction between the groundwater and certain fluoride-rich minerals could explain the variation in fluoride concentration observed in the recharge and flow-through area of northern Mt. Meru, while in the discharge area, evaporation and exchange capacity made a greater contribution to the fluoride presence in the surface- and groundwater. The groundwater emerging from phonolite had a low content of both alkaline cations and fluoride, due to the relatively 
low reactivity of silicate. The phonolite hosted-aquifers are highly fractured, and are thus highly permeable (Ghiglieri et al., 2010). Water carried through a scoriaceous, highly permeable, basalt aquifer showed an even lower concentration of fluoride and alkaline cations. The elevated fluoride content of lahar-hosted groundwater, on the other hand, derived from both the high permeability of the rock and the contribution of zeolites which both contain a high level of exchangeable cations and have an ash matrix. Volcanic ash is a major source of fluoride release. The dissolution process (and particularly, the cyclic dissolution/precipitation of the fluoriderich trona which occurs as a seasonal encrustation in lowlying river valleys and ponds at lake margins), on the other hand, is an important factor behind the high fluoride content of the saturated superficial sediments. Hydrothermal springs, such as those which feed into the Ngarenanyuki river, make a further contribution to the level of fluoride pollution in the groundwater.

The geo-lithological characteristics of an aquifer, the recharge altitude and the residence time of the groundwater together determine the extent of the water ionic charge. Three major types of aquifer can be distinguished. The first is the high altitude phonolite-hosted aquifer, in which high transmissivity, high elevation and low temperature $\left(16^{\circ} \mathrm{C}\right)$ together serve to inhibit the rate of dissolution of both mineral and glassy groundmass, giving rise to a low fluoride groundwater. The second is the sedimentary (lahar and alluvial/lacustrine) hosted aquifer, frequently found at the base of Mt. Meru. Their relatively low altitude means that high temperatures prevail near the surface, favouring the precipitation of $\mathrm{CaCO}_{3}$, and thereby inhibiting the precipitation of $\mathrm{CaF}_{2}$ and producing a higher fluoride content. The occurrence of CEC-endowed neoformed phases, along with the magadi, enhances the seasonal cycle of fluoride entrapment/release. Finally, the buried basalt hosted type of aquifer, associated with an elevated recharge area, shows a high transmissivity (Ghiglieri et al., 2010) which is optimal for maintaining a low ionic content. Infiltration into these aquifers occurs under rather cool conditions, the water tends to have a low residence time and no feeding occurs from the flatter areas where calcrete formation can deplete the level of calcium and thereby raise the fluoride concentration in the water.

Acknowledgements. This research was done as part of a project funded by OIKOS Institute (Italy), Charity and Defence of Nature Fund (private foundation) and Sardinia local Government (Italy) (Regional Law 19/96: cooperation with developing countries). Thanks are due also to Fondazione Banco di Sardegna for the financial support to D. Pittalis. For the purpose of the research, thanks are due to OIKOS EAST-AFRICA for technical and logistic support in Tanzania.

Edited by: Y. Fan

\section{References}

APHA: Standard methods for examination of water and wastewater. America Pubblic Health Association, Washington D.C.-APHA, 1992.

Balcone-Boissard, H., Baker, D. R., Villemant, B., and Boudon, G.: $\mathrm{F}$ and $\mathrm{Cl}$ diffusion in phonolitic melts: Influence of the $\mathrm{Na} / \mathrm{K}$ ratio, Chem. Geol., 263, 89-98, 2009.

Bruker AXS: Diffracplus Evaluation package EVA 14, Release 15 July 2008, Bruker AXS GmbH Karlsruhe, Germany, 2008.

Clarke, M. C. G., Woodhall, D. G., Allen, D., and Darling, G.: Geological, volcanological and hydrological controls on the occurrence of geothermal activity in the area surrounding Lake Naivasha, Kenya, Ministry of Energy Report, Nairobi, Kenya, 138 pp., 1990.

Coleman, M. C., Shepherd, T. J., Durham, J. J., Rouse, J. D., and Moore, G. R.: Reduction of water with zinc for hydrogen isotope analysis, Anal. Chem., 54, 993-995, 1982.

Craig H.: Standards for reporting concentrations of deuterium and oxygen-18 in natural waters, Science, 133, 1833-1834, 1961.

Davies, T. C.: Chemistry and pollution of natural waters in western Kenya, J. Afr. Earth Sci., 23, 547-563, 1996.

Deocampo, D. M.: Hydrogeochemistry in the Ngorongoro Crater, Tanzania, and implications for land use in a World Heritage Site, Appl. Geochem., 19, 755-767, 2003.

Dettman, D. L., Palacios-Fest, M. R., Nkotagu, H., and Cohen, A. S.: Paleolimnological investigations of anthropogenic environmental change in Lake Tanganyka: VII. Carbonate isotope geochemistry as a record of riverine runo?, J. Paleolimnol., 34, 93$105,2005$.

Epstein, S. and Mayeda, T.: Variation of O18 content of waters from natural sources, Geochim. Cosmochim. Ac., 4, 213-224, 1953.

Fuge, R. and Andrews, M. J.: Fluoride in the UK environment, Environ. Geochem. Health, 10, 96-104, 1988.

Gao, X., Wang, Y., Li, Y., and Guo, Q.: Enrichment of fluoride in groundwater under the impact of saline water intrusion at the salt lake area of Yuncheng basin, northern China, Environ. Geol., 53, 795-803, doi:10.1007/s00254-007-0692-z, 2007.

Ghiglieri, G., Balia, R., Oggiano, G., Ardau, F., and Pittalis, D.: Hydrogeological and geophysical investigations for groundwater in the Arumeru District (Northern Tanzania), Presentation at the 84 National Meeting of the Italian Geologic Society, Sassari, 1517 September 2008 (Conference Proceedings, 431-433, Vol. 2), 2008.

Ghiglieri, G., Balia, R., Oggiano, G., and Pittalis, D.: Prospecting for safe (low fluoride) groundwater in the Eastern African Rift: the Arumeru District (Northern Tanzania), Hydrol. Earth Syst. Sci., 14, 1081-1091, doi:10.5194/hess-14-1081-2010, 2010.

Hijmans, R. J., Cameron, S. E, Parra, J. L., Jones, P. G., and Jarvis, A.: Very high resolution interpolated climate surfaces for global land areas, Int. J. Climatol., 25, 1965-1978, 2005.

ICDD: International Centre for Diffraction Data. PDF-2. Release 2003, Newton Square, Pennsylvania, USA, 2003.

Jalali, M.: Hydrochemical identification of groundwater resources and their changes under the impacts of human activity in the Chah Basin in western Iran, Environ. Monit. Assess., 130, 347364, 2007.

Jones, B. F., Eugster, H. P., and Rettig, S. L.: Hydrochemistry of the Lake Magadi basin, Kenya, Geochim. Cosmochim. Ac., 41, 53-72, 1977. 
Kilham, P. and Hecky, R. E.: Fluoride: Geochemical and Ecological significance in East African waters and sediments, November, Limnol. Oceanogr., 18, 932-945, 1973.

Nanyaro, J. T., Aswathanarayana, U., Mungure, J. S., and Lahermo, P. W.: A geochemical model for the abnormal fluoride concentrations in waters in parts of northern Tanzania, J. African Earth Sci., 2, 129-140, 1984.

Nielsen, J. M.: East African magadi (trona): fluoride concentration and mineralogical composition, J. Afr. Earth Sci., 29, 423-428, 1999.

Parkhurst, D. L. and Appelo, C. A. J.: User's guide to PHREEQC (version 2) - a computer program for speciation, batch-reaction, one-dimensional transport, and inverse geochemical calculations. United States Geological Survey, Water Resources Investigations Report 99-4259, Washington, p. 326, 1999.
Sebag, D., Verrecchia, E. P., Leed, S. J., and Durand, A.: The natural hydrous sodium silicates from the northern bank of Lake Chad: occurrance, petrology and genesis, Sediment. Geol., 139, 15-31, 2001.

Signorelli, S., Vaggelli, G., and Romano, C.: Pre-eruptive volatile $\left(\mathrm{H}_{2} \mathrm{O}, \mathrm{F}, \mathrm{Cl}\right.$ and $\left.\mathrm{S}\right)$ contents of phonolitic magmas feeding the 3550-year old Avellino eruption from Vesuvius, southern Italy, J. Volcanol. Geoth. Res., 93, 237-256, 1999.

Su, Y. H., Feng, Q., Zhu, G. F., Si, J. H., and Zhang, Y. W.: Identification and Evolution of Groundwater Chemistry in the Ejin Sub-Basin of the Heihe River, Northwest China, Pedosphere, 17, 331-342, doi:10.1016/S1002-0160(07)60040-X, June 2007.

US DEPARTMENT OF ENERGY, Environmental Measurements Laboratory: Tritium in water - acid electrolysis $-3 \mathrm{H}-01-\mathrm{RC}$, HASL - 300, 28th Edition, 1997. 